\title{
Stellungnahme zu den medizinisch-ethischen Richtlinien «Betreuung von Patienten am Lebensende»
}

Ethikforum Inselspital Bern

Korrespondenz:

Dr. theol. Plasch Spescha Büro Katholische Kapelle Inselspital

CH-3010 Bern

Tel. 0316322846

E-Mail: plasch.spescha@insel.ch
Das Ethikforum des Berner Inselspitals durfte sich am 23. Februar 2004 durch Herrn Dr. theol. Markus Zimmermann aus erster Hand über die neuen medizinisch-ethischen Richtlinien informieren zu lassen. Unter anderem verwies er auf das Interesse an Stellungnahmen seitens der Adressaten hin. Das Ethikforum des Inselspitals hat dieses Interesse erfreut zur Kenntnis genommen und nimmt diese Möglichkeit mit der vorliegenden Stellungnahme gerne wahr.

\section{Zustimmung}

Insgesamt sind wir mit den Richtlinien zur Betreuung von Patienten am Lebensende einverstanden. Besonders hervorheben möchten wir dabei fünf Punkte:

a) die sprachliche Einfachheit und der fast durchgängige Verzicht auf fachsprachlichen Jargon, sowohl medizinisch als auch ethisch;

b) der Argumentationsstil ist sehr differenzierend und abwägend. Hier kommt viel von der alten aristotelischen Tugend der Klugheit zum Zuge;

c) die klare Zustimmung zum Instrument der Patientenverfügung. Sie wird definitiv nicht mehr als Kampfinstrument der sozialen Bewegung für humanes Sterben abgelehnt, sondern als wichtiges Informationsmittel und als nützliche Entscheidungshilfe für eine patientengerechte Behandlung verstanden;

d) das klare Plädoyer für palliative Behandlung und Betreuung;

e) die Berücksichtigung der ärztlichen Gewissensentscheidung im Umgang mit dem Sterben, ausdrücklich in der Beihilfe zum Suizid. Damit wird nicht nur dem Menschenrecht der Gewissensfreiheit im Berufsethos Rechnung getragen, sondern auch der ethischen Pluralität in der Ärzteschaft. Auf diese Weise wird die ethische Pluralität in der heutigen Gesellschaft im ärztlichen Berufsethos angemessen abgebildet.

\section{Kritik und Rückfragen}

$\mathrm{Zu}$ fragen und zu diskutieren geben verschiedene Punkte. Hervorheben möchten wir die folgenden:

\section{Zu 1: Geltungsbereich}

Der Kommentar bringt neu den Begriff «Terminalphase» ein. Er bezieht ihn auf einen Zeitraum «bis zu einem Jahr oder länger» und macht einen Unterschied zu «Lebensende». Unklar wird dabei, welches nun der weitere und welches der engere Begriff ist. Nichtmediziner assoziieren mit «Terminalphase» in der Regel keine Zeiträume, welche Monate oder gar mehr als ein Jahr betreffen, sondern die letzten, wenn nicht gar allerletzten Lebenstage und -stunden. Die Frage ist, ob es hier die Verknüpfung mit dem typisch medizinischen Ausdruck «terminal» überhaupt braucht.

Beim Geltungsbereich stellt sich darüber hinaus eine Grundsatzfrage. In den Richtlinien wird das Sterben ausschliesslich oder mindestens prioritär als medizinisches Thema betrachtet. In der Realität ist es jedoch vor allem auch ein lebensgeschichtliches und soziales Geschehen. Unter dieser Voraussetzung müsste der Einsatzpunkt für die Rede vom Lebensende anders definiert werden. Es ist dann nicht allein die medizinisch erfasste Realität, sondern das Reden und Schweigen der Betroffenen und Beteiligten über ein mögliches Sterben und den Tod. Die seelsorgliche Erfahrung zeigt, dass bei den Betroffenen und ihrem Umfeld Sterben und Tod nicht selten schon eine gedankliche und sprachliche Realität und reale Möglichkeit ist, wenn dies auf ärztlicher Seite noch nicht der Fall ist und manchmal noch weit von sich gewiesen wird.

Eine besondere Rolle bei der Thematisierung des Sterbens spielen die Entscheidungsprozesse im Übergang von einer kurativen $\mathrm{zu}$ einer palliativen Behandlung, Betreuung und Begleitung. In diesem Zusammenhang gehört die Präsenz des Themas Lebensende 
schon definitionsgemäss zur medizinischen Realität.

Beide Überlegungen laufen auf einen doppelten Wunsch hinaus: im Titel der Richtlinien $\mathrm{zu}$ verdeutlichen, dass es um die ärztliche Betreuung am Lebensende geht, und den Geltungsbereich in die Tatsache einzubetten, dass das Lebensende eine lebensgeschichtliche und soziale Realität ist, in welcher die medizinische Seite nur ein Element und die ärztliche Aufgabe eine von mehreren ist.

\section{Zu 2.2: Beizug von klinischen Ethik- kommissionen}

- Ethikkommissionen sind in der Regel ein sehr schwerfälliges Instrument. Häufig wird die Bezeichnung für Bewilligungsinstanzen für medizinische Forschungsprojekte verwendet. Seltener werden unter dieser Bezeichnung Gremien verstanden, welche sich mit klinischen Einzelfällen beschäftigen. Im Inselspital suchen wir den Weg über einen ethischen Beratungsdienst bzw. über das Ethikkonsilium. Dieses hat zwei Formen: als Beratung durch eine ethische Fachperson und als Ethikgespräch, in welchem die Beteiligten nach einer Entscheidung suchen, welche gemeinsam verantwortet werden kann. Beiden Formen ist gemeinsam, dass es darum geht, ethische Expertise in die Entscheidungsprozesse vor Ort und im Einzelfall zu integrieren.

- Die Erfahrung zeigt zudem, dass die ethische Beratung nicht nur in Ziff. 2, sondern in allen Bereichen, welche die Richtlinien abdecken, hilfreich und nützlich sein kann.

\section{Zu 3.2: Behandlungsverzicht oder -abbruch}

Es gibt Situationen, in welchen diese nicht nur gerechtfertigt sein können, sondern sind.

\section{Zu 4.1: Beihilfe zum Suizid}

In diesem Zusammenhang stellt sich zuerst und vordringlich die Frage nach der Bedeutung des schwierigen Sterbens. Die Lebensendediskussion ist stark geprägt vom Ideal des sanften Sterbens, des ruhigen und schmerzlosen Einschlafens. Ist dies nicht gegeben, muss es medizinisch hergestellt werden. Das kommt einer Idealisierung des Sterbens gleich und zeugt von Realitätsverlust. Dabei wird völlig ausgeblendet, dass Sterben auch eine Identitätskrise sein kann und wie jede andere menschliche Identitätskrise ihre je eigenen Leidens- und Reifungsmöglichkeiten beinhaltet. Diese sollten nicht leichtfertig vergeben werden, auch wenn Leiden in der christlichen Geschichte allzuoft als Erlösung und Läuterung hingestellt und missbraucht wurde.

Die Forderung nach Beihilfe zum Suizid und dies ist eine weitere grundsätzliche Bemerkung - bewegt sich im Kontext des Rechts auf Selbstbestimmung. Weil damit der wohl höchste gesellschaftliche Wert, die individuelle Freiheit, angesprochen ist, hat sie eine hohe gesellschaftliche Plausibilität. Dabei wird übersehen, dass dies im Kern eine «halbierte» Selbstbestimmung ist. Ausgeblendet wird, dass auch die Entscheidung, auf den Tod zu warten, Ausdruck von Selbstbestimmung und Freiheit ist. Dieser Aspekt, den man das Recht auf Warten nennen kann, müsste ausdrücklich genannt werden und in das ärztliche Ethos integriert werden. Schmerzen bekämpfen und Leiden lindern im Kontext des Wartens auf den Tod und im Kontext der Beihilfe zum Suizid gehorchen einer anderen inneren Logik. Wo genau die Unterschiede liegen, wäre wohl noch herauszuarbeiten.

Eine weitere Frage ist: Müsste nicht so klar wie möglich formuliert werden, dass die ärztliche Rolle beim Sterben in den Hintergrund tritt und primär andere Fähigkeiten und Fertigkeiten gefragt sind.

Zum Text selber:

- Auch wenn die Beihilfe zum Suizid «nicht Teil der ärztlichen Tätigkeit» ist, bleibt ein spezielles berufsspezifisches Problem weiterhin bestehen. Das wichtigste Mittel, welches heute in der Schweiz dafür eingesetzt wird, ist rezeptpflichtig. Das Ausstellen des entsprechenden Rezepts ist aber eine ärztliche Handlung.

- Die Beihilfe zum Suizid gilt einerseits nicht als Teil der ärztlichen Tätigkeit anderseits als mögliche Gewissenshandlung. Nach der ersten Aussage würde man eigentlich eine Ablehnung erwarten, weil sie kategorisch formuliert ist. Gewissensentscheidungen in einem kategorischen Kontext sind ethisch gesehen immer ein Verstoss oder aber - und diese wäre eine andere Sicht - sie machen auf ein Ungenügen des herrschenden Ethos aufmerksam. Ist letzteres der Fall, dann müsste die kategorische Aussage zurückgenommen oder mit einem anderen Bezugspunkt formuliert werden. Eine Möglichkeit wäre, zu sagen: die Beihilfe zum Suizid «ist keine ärztliche Pflicht». Auf diese Weise könnte ohne Wenn und Aber zum Ausdruck gebracht werden, dass kein Arzt und keine Ärztin dazu genötigt werden kann und dass dem auch kein Recht auf seiten der Sterbenden entspricht. 


\section{Zu 4.2: Tötung auf Verlangen}

- Ist die Identifikation dieses StGB-Artikels mit aktiver Sterbehilfe richtig, wenn anschliessend allein vom urteilsfähigen Patienten die Rede ist? Die grossen Probleme stellen sich eigentlich erst, wenn der Patient nicht mehr urteilsfähig ist. Wenn zudem eine entsprechend formulierte Patientenverfügung vorhanden ist, wird das Problem noch schwieriger. Die dann auftretenden Gewissenskonflikte gehen tief. Sie sind geprägt von typischsten Merkmal von Gewissenskonflikten, nämlich der Frage nach der bewussten und überlegten Verletzung von geltenden Normen und Gesetzen - weil man nicht anders kann, will man die Integrität der eigenen Person bewahren. Von daher stellt sich die Grundsatzfrage, ob medizinischethische Richtlinien sich ausschliesslich an den gegebenen strafrechtlichen Regelungen orientieren sollen oder ob sie nicht auch auf einen gesetzgeberischen Handlungsbedarf aufmerksam machen sollten. Wie auch immer diese Frage beantwortet wird, in ethischer Sicht liegt in der genannten Situation auf jeden Fall ein Gewissenskonflikt vor.

- Zudem stellt sich die Frage, ob in medizinisch-ethischen Richtlinien der rechtliche Begriff «Urteilsfähigkeit» übernommen werden soll oder ob nicht ein anderer Begriff gewählt werden sollte, z.B. Verhandlungsfähigkeit.

\section{Neue Probleme und Fragen}

Im Zusammenhang mit dem Lebensende sind in den letzten Jahren neue Handlungsmöglichkeiten bewusst geworden. Sie werden bisher nur wenig beachtet, doch können bei ihnen ärztliches Wissen und Entscheiden eine grosse Rolle spielen. Deshalb stellt sich die Frage, ob die Richtlinien nicht ausdrücklich darauf eingehen sollten.

Es sind vor allem zwei solche Situationen, an welche zu denken ist: Es gibt z.B. Patientinnen und Patienten, welche dank einer guten und in der Regel sehr komplexen Medikation ein weitgehend selbständiges und gutes Leben führen können. Nach langen Jahren eines Lebens, welches dank der Medikamente geführt werden konnte, kristallisiert sich der Wunsch und Wille heraus, es zu beenden. Ein möglicher Weg dazu wäre, die Medikamente abzusetzen. Um dies ohne zusätzliche Schmerzen und grosse Beeinträchtigungen tun zu können, bräuchten sie jedoch fachliches Wissen. Eine andere Handlungsmöglichkeit am Lebensende ist das Sterbefasten. Dabei geht es um Menschen, welche sich entscheiden, auf Nahrung und Trinken zu verzichten. In den bekanntgewordenen Beispielen geht es um Menschen in hohem Alter, welche schon lange auf den Tod warten und auf ein zufriedenes und sinnvolles Leben zurückblicken.

In beiden Situationen geht es um Formen von Suizid und in beiden Situationen spielen medizinische Aspekte und ärztliches Wissen eine Rolle. Die Frage ist dann, ob eine ärztliche Begleitung mit dem Berufsethos vereinbar ist und wenn ja, was das heissen würde. 